\title{
ANATOMIA DO PLANO INTERMUSCULAR LOMBAR ENTRE OS MÚSCULOS MULTÍFIDUS E LONGUÍSSIMO E PLANEJAMENTO PRÉ-OPERATÓRIO COM IMAGENS DE RESSONÂNCIA NUCLEAR MAGNÉTICA PARA ARTRODESES LOMBARES MINIMAMENTE INVASIVAS
}

\author{
ANATOMIC FEATURES OF THE INTERMUSCULAR LUMBAR PLANE BETWEEN THE MULTIFI- \\ DUS AND THE LONGISSIMUS MUSCLES AND PREOPERATIVE PLANNING WITH MRI IMAGES \\ FOR MINIMALLY INVASIVE LUMBAR FUSION
}

ANATOMÍA DEL PLANO LUMBAR INTERMUSCULAR, ENTRE EL MÚSCULO MULTÍFIDO Y EL MÚSCULO LONGÍSIMO, Y EL PLANEAMIENTO PREOPERATORIO CON IMÁGENES DE RESONANCIA NUCLEAR MAGNÉTICA PARA ARTRODESIS LUMBARES MIIINIMAMENTE INVASORAS

Fernando Schmidt ${ }^{1}$, Thiago Soares dos Santos ${ }^{2}$, Marcelo Maroues Soares ${ }^{3}$

\begin{abstract}
RESUMO
Objetivo: Revisar a anatomia da musculatura lombar posterior, demonstrá-la no plano axial da ressonância magnética e medir a distância da linha média até o plano intermuscular entre os músculos multífidus e longuíssimo nos níveis L3/L4, L4/L5 e L5/S1. Métodos: Através do programa OSIRIX para Mac foram realizadas medidas em 50 pacientes adultos, 25 homens e 25 mulheres. Mensuramos a distância bilateralmente nos níveis lombares inferiores. Resultados: A distância média foi de 2,42 cm em L3/L4, de 3,13 cm em L4/L5 e de 3,77 cm em L5/S1, quando não separamos os sexos. Houve um aumento da distância média no sentido craniocaudal nos níveis lombares inferiores e quando comparamos os sexos não houve diferença estatisticamente significativa nos níveis L4/L5 e L5/S1. Conclusão: Concluímos que o exame de ressonância magnética permite mensurar a distância da linha média até o plano intermuscular entre o multífidus e o longuíssimo e consideramos importante para o planejamento pré-operatório dos procedimentos minimamente invasivos.
\end{abstract}

Descritores: Ressonância magnética; Medidas; Anatomia; Procedimentos cirúrgicos minimamente invasivos; Artrodese.

\begin{abstract}
Objective: The purpose of this work is to review the posterior lumbar muscle anatomy, evince the correlation between the anatomy in the axial plane of the MRI and measure the distance from the medium line to the intermuscular plane, between the multifidus and the longissimus in the lower lumbar levels. Methods: Using the OSIRIX program for Mac, bilateral measurements of the distance from the medium line to the intermuscular plane between the multifidus and longissimus muscles have been performed in the L3/L4, L4/L5 and L5/S1 levels, in 50 adult patients divided equally by gender. Results: This study brings to the conclusion that there is an average distance of $2.42 \mathrm{~cm}$ in $\mathrm{L} 3 / \mathrm{L} 4$, of $3.13 \mathrm{~cm}$ in L4/L5 and of $3.77 \mathrm{~cm}$ in L5/S1 when not separated by gender. The average distance increases in the craniocaudal direction in the lower lumbar levels and there is no statistically significant difference toward the gender in levels L4/L5 and L5/S1. Conclusion: We conclude that the MRI allows the surgeon to measure the distance from the midline until the intermuscular plane between the multifidus and longissimus and consider it important for the preoperative planning of minimally invasive approaches.
\end{abstract}

Keywords: Magnetic resonance imaging; Measures; Anatomy; Surgical procedures, minimally invasive; Arthrodesis.

\section{RESUMEN}

Objetivos: Revisar la anatomía de la musculatura lumbar posterior, demostra la en el plano axial de la resonancia magnética y media la distancia desde la línea media al plano intermuscular, entre el músculo multífids y el longísimo, en los niveles de L3/L4, L4/L5 y L5/S1. Metodos: Mediante el programa Osirix para Mac se hicieron medicionen en 50 pacientes adultos, 25 hombres y 25 mujeres. Se midió la distancia bilateralmente en los niveles lumbares inferiores. Resultados: La distancia media fue 2,42 cm en L3/L4, 3,13 cm en L4/L5 y 3,77 cm en L5/S1 cuando no separamos por sexos. Hubo un aumento de la distancia media en la dirección craneocaudal en los niveles lumbares inferiores y al comparar los sexos no hubo diferencia estadísticamente significativa en los niveles L4/L5 y L5/S1. Conclusion: Concluimos que el examen de resonancia magnética nos permite medir la distancia de la linea media hasta el plano intermuscular, entre el multífido y el longísimos, y nosotros lo consideramos importante para ea planeamientn preoperatoria de los procedimientos minimamente invasores.

Descriptores: Resonancia magnética; Medidas; Anatomia; Procedimientos quirúrgicos mínimamente invasores; Artrodesis.

1. Médico Neurocirurgião do Serviço de Coluna do Hospital Regina de Novo Hamburgo - RS, Brasil.

2. Médico Ortopedista do Serviço de Coluna do Hospital Regina de Novo Hamburgo - RS, Brasil.

3. Professor de Anatomia da Feevale de Novo Hamburgo - RS, Brasil.

Trabalho realizado no Departamento de Anatomia da Feevale e no Serviço de Coluna do Hospital Regina de Novo Hamburgo, RS. Brasil.

Correspondência: Av. Mauricio Cardoso, 833, sala 310, Hamburgo Velho. Novo Hamburgo. Brasil. CEP 93510250. E-mail: fschmidt.nho@terra.com.br 


\section{INTRODUÇÃO}

A abordagem lateral paramediana para coluna toracolombar vem sendo desenvolvida há anos por vários autores. Em 1936 Mathieu e Dermileau ${ }^{1}$ foram os primeiros a descreverem uma abordagem lateral a musculatura sacroespinhal. Em 1953 Watkins ${ }^{2}$ descreveu uma abordagem lateral entre o eretor da espinha (iliocostal) e o músculo quadrado lombar, necessitando osteotomia do ilíaco para rebater a musculatura. Wiltse e Hutchinson ${ }^{3}$, e Witse e Spencer ${ }^{4}$ mais tarde, descreveram uma abordagem lateral entre o músculo multifidus e o músculo longuissimo. Esse acesso utiliza um plano natural no sentido longitudinal entre esses dois músculos ${ }^{3}$

O desenvolvimento e utilização dessas vias procuram minimizar o trauma aos tecidos. A exploração anatômica da região lombar posterior revela uma complexa arquitetura envolvendo fáscias, músculos e componentes neurovasculares ${ }^{5}$. A compreensão tridimensional da anatomia dos grupos musculares e individualização das fáscias define os limites da área de trabalho diminuindo o dano dos mesmos. Esse acesso permite alcançar os mesmos objetivos cirúrgicos da abordagem convencional mediana, porém preservando a função muscular e neurovascular melhorando assim os resultados clínicos.

Na identificação pré-operatória para as abordagens paramedianas lombares intermusculares entre o músculo multífidus e o músculo longuíssimo é fundamental o conhecimento anatômico deste plano pelo cirurgião. Revisamos os aspectos mais importantes da anatomia posterior da coluna lombar, identificando pela dissecção em cadáveres os pontos e limites topográficos da musculatura, fáscias, aponeuroses e estruturas neurovasculares. O objetivo deste trabalho é revisar a anatomia posterior da coluna lombar, identificar através do exame de ressonância nuclear magnética o plano intermuscular entre os músculos multífidus e longuíssimo e medir a sua distância da linha média, visando melhorar a compreensão e facilitar a execução da abordagem intermuscular lombar.

\section{MATERIAIS E MÉTODOS}

Foram selecionados 50 pacientes adultos com queixa de lombalgia, 25 sexo masculino e 25 sexo feminino. Excluímos pacientes com patologia tumoral, infecciosa e cirurgia prévia. Foram utilizados 50 exames consecutivos de ressonância nuclear magnética da coluna lombosacra em aparelho de 1.5 Tesla.

Utilizamos o programa OSIRIX para Mac realizar medidas bilaterais nos cortes axiais em T2 dos níveis L3/L4, L4/L5 e L5/S1. A medida foi feita através de uma linha traçada na altura do terço superior da faceta articular superior, local de inserção do músculo multífidus, tendo como ponto de referência na linha média a ponta da apófise espinhosa e como ponto de referência lateral o espaço intermuscular entre o músculo multífidus e longuíssimo (Figura 1). Foram realizadas 50 medidas para cada nível em cada um dos sexos.

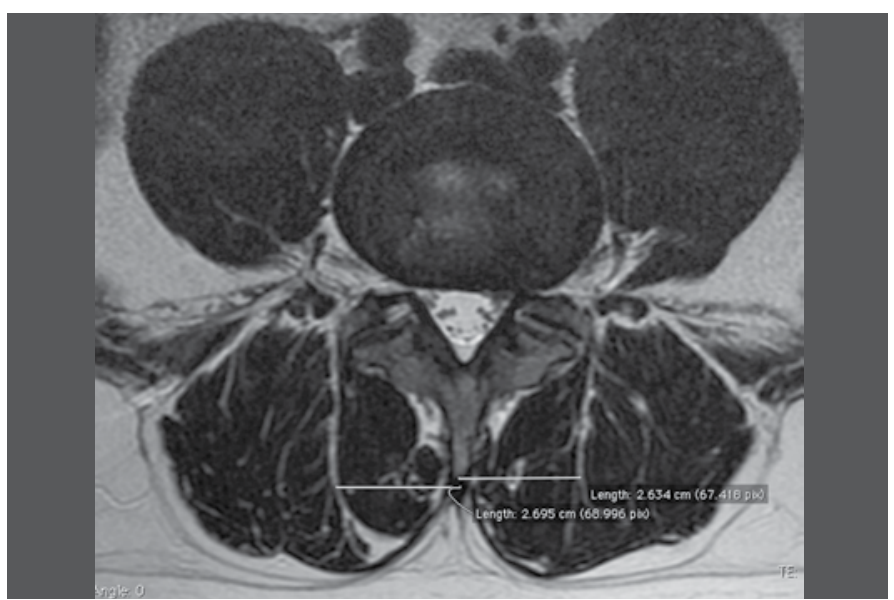

Figura 1. Medida em L4/L5
Os resultados foram gerados pelo software SPSS (Statistical Package for Social Sciences), versão 17.0. Foram feitas estatísticas descritivas (para cálculo da média e desvio-padrão), testes comparativos (Teste $t$ ) entre médias, separadas por sexo, e foi usado o teste ANOVA para amostras independentes comparando os 3 níveis, sem separação por sexo.

\section{Anatomia da muscultura lombar posterior}

Segundo estudo de Macintosh et al. ${ }^{6}$ e de acordo com nossas dissecções em cadáveres, encontramos na porção posterior às apófises transversas, duas grandes massas musculares, medialmente o músculo multífidus e lateralmente o músculo eretor da espinha, este constituído por dois músculos, o longuíssimo torácico e o iliocostal lombar. O músculo multífidus apresenta um complexo de feixes que se inserem nos processos mamilares das facetas articulares superiores e se originam nas apófises espinhosas e lâminas vertebrais lombares. O músculo longuíssimo torácico parte lombar tem origem nos processos acessórios das facetas articulares superiores e se insere na crista ilíaca. Dessa forma a arquitetura destes dois músculos cria um plano de clivagem natural a cerca da borda lateral da faceta articular superior. Para as abordagens minimamente invasivas é importante identificar este plano e após a abordagem anatômica através deste corredor cirúrgico é possível expor o complexo articular, a base do pedículo e a porção posterior das apófises transversas permitindo inúmeros procedimentos cirúrgicos como descompressão radicular, discectomia, TLIF e instrumentação com parafusos pediculares

Nas abordagens cirúrgicas, após incisão da pele e tecido celular subcutâneo, encontramos a fáscia do músculo grande dorsal. Abaixo dessa fáscia existe a aponeurose do músculo eretor da espinha que é formada a partir do músculo longuíssimo torácico parte torácica e iliocostal lombar parte torácica, sem inserção lateral nas vértebras lombares, mas apenas no sacro e osso ilíaco. Esta aponeurose é formada por uma porção medial que cobre os músculos multífidus e longuíssimo torácico parte lombar e uma parte lateral que cobre o músculo iliocostal lombar parte lombar. Uma pequena depressão identificada nas peças anatômicas e nos exames de ressonância magnética mostra o limite entre estas duas porções da fáscia do músculo eretor da espinha e corresponde ao espaço intermuscular entre os músculos longuíssimo e iliocostal. Para atingir cirurgicamente o espaço intermuscular entre o músculo multífidus e longuíssimo não temos um reparo anatômico na fáscia, mas podemos obtê-lo através da medida pré-operatória que pode ser feita no exame de ressonância nuclear magnética e através da marcação desse plano na pele sob fluoroscopia, uma vez que este plano esta imediatamente lateral ao complexo articular facetário lombar.

\section{RESULTADOS}

Quando comparamos as medidas entre os sexos encontramos uma média no nível L3/L4 para mulheres de $2,35 \mathrm{~cm}$ e para os homens foi de $2,49 \mathrm{~cm}$. Como o valor da significância foi de 0,019 rejeitamos a hipótese nula, ou seja, o nível L3/L4 se difere por sexo $(t=2,394, p=0,019)$ ou $(t=2,394, p<0,05)$. A média do nível $L 4 / L 5$ para mulheres foi de $3,07 \mathrm{~cm}$ e para homens foi de 3,20. Como o valor da significância foi de 0,090 aceitamos a hipótese nula, ou seja, o nível L4/L5 não se difere por sexo $(t=-1,717, p=0,090)$ ou $(t=-1,717, p>0,05)$. A média do nível L5/S1 para mulheres foi de $3,84 \mathrm{~cm}$ e para homens foi de $3,70 \mathrm{~cm}$. Como o valor da significância foi de 0,091 aceitamos a hipótese nula, ou seja, o nível L5/S1 não se difere por sexo $(t=-1,709, p=0,091)$ ou $(t=-1,709, p>0,05)$. Para estas analises aplicamos o T-test e, portanto, houve diferença estatisticamente significativa apenas no nível L3/L4 entre sexos, o que para nos não representou mudança no planejamento préoperatório (Tabelas 1 e 2).

Na análise sem a distinção dos sexos encontramos uma média para os níveis $L 3 / L 4$ igual a 2,42 cm com desvio padrão de 0,30 , a média nos níveis L4/L5 foi igual a $3,13 \mathrm{~cm}$ com desvio padrão de 
0,36 e a média nos níveis $L 5 / S 1$ foi igual a $3,77 \mathrm{~cm}$ com desvio padrão de 0,42. Observando as significâncias apresentadas, sempre que forem menores do que 0,05 podemos dizer que os grupos se diferem. De acordo com teste ANOVA há diferença estatisticamente significativa pelo menos entre 2 dos 3 grupos $(F=337,257$, $\mathrm{p}=0,000)$ ou $(\mathrm{F}=337,257, \mathrm{p}<0,05)$. Percebemos então que os níveis se diferem significativamente e fica evidente que há um aumento da distancia media no sentido craniocaudal (Tabelas 3 e 4).

Tabela 1. Média por nível no sexo feminino.

\begin{tabular}{c|c|c|c|c}
\hline Nível & Mínimo & Máximo & Média & Desvio \\
\hline L3/L4 & 1.68 & 2.91 & 2.354 & 0.246 \\
\hline L4/L5 & 2.5 & 3.9 & 3.075 & 0.288 \\
\hline L5/S1 & 2.6 & 5 & 3.845 & 0.399 \\
\hline
\end{tabular}

Tabela 2. Média por nível no sexo masculino.

\begin{tabular}{c|c|c|c|c}
\hline Nível & Mínimo & Máximo & Média & Desvio \\
\hline L3/L4 & 1.78 & 3 & 2.495 & 0.334 \\
\hline L4/L5 & 2.2 & 3.9 & 3.2 & 0.428 \\
\hline L5/S1 & 2.6 & 4.4 & 3.702 & 0.436 \\
\hline
\end{tabular}

Tabela 3. Media por nível em ambos sexos.

\begin{tabular}{c|c|c|c|c}
\hline Nível & Mínimo & Máximo & Média & Desvio \\
\hline L3/L4 & 1.68 & 3 & 2.424 & 0.301 \\
\hline L4/L5 & 2.2 & 3.9 & 3.137 & 0.368 \\
\hline L5/S1 & 2.6 & 5 & 3.774 & 0.422 \\
\hline
\end{tabular}

\section{DISCUSSÃO}

Nos últimos anos tem havido uma discussão a cerca das causas de lombalgia pós cirúrgicas na coluna lombar. Muitos autores vêem estudando os efeitos adversos causados pela extensa dissecção e retração muscular que normalmente ocorrem nos procedimentos convencionais abordados pela linha media ${ }^{7}$. Kawaguchi et al..$^{89}$ analisaram os efeitos da pressão na musculatura paraespinhosa causados pelos retratores durante os procedimentos lombar por via convencional. Eles observaram elevados níveis de CPK MM, indicador de lesão muscular, e determinaram que estavam diretamente relacionados com pressão e duração da retração muscular. Esses resultados foram ao encontro do estudo de Gejo et al. ${ }^{10}$ que examinaram imagens de RNM pós operatórias e a força da musculatura do tronco em 80 pacientes submetidos a cirurgia lombar. Eles concluíram que o dano muscular está diretamente relacionado ao tempo de retração e observaram que a incidência de lombalgia era maior nos pacientes com maior tempo cirúrgico. Styf e Willen ${ }^{11}$ determinaram a relação entre pressão durante a retração muscular e a isquemia. Mayer et al. ${ }^{12}$ compararam a força muscular dos pacientes submetidos a fusão e a discectomia e observaram déficit de força maior nos pacientes com fusão. Rantanem et al. ${ }^{13}$ concluíram que os pacientes com piores resultados clínicos apresentavam maior lesão na musculatura paraespinhais.

Técnicas minimamente invasivas tem sido o "gold standard" para o tratamento de diversas patologias em várias especialidades cirúrgicas ${ }^{7}$. Não diferente, a cirurgia da coluna vertebral vem desenvolvendo técnicas para minimizar os danos e melhorar os resultados.

Segundo Hoh et al..$^{5}$ as abordagens intermusculares, paramedianas lombares, entre o músculo multifidus e o longuíssimo utilizam um plano de clivagem natural que permite a preservação das suas fibras musculares e suas inserções, bem como, sua função, diminuindo a lesão dos mesmos, o que evidenciamos neste estudo. O objetivo da preservação da musculatura e das estruturas
Tabela 4. Medidas sexo feminino e masculino

\begin{tabular}{|c|c|c|c|c|c|c|}
\hline \multirow[t]{3}{*}{ SEXO } & \multicolumn{2}{|c|}{ NÍVEL } & \multicolumn{2}{|c|}{ NÍVEL } & \multicolumn{2}{|c|}{ NÍVEL } \\
\hline & \multicolumn{2}{|c|}{ L3/L4 } & \multicolumn{2}{|c|}{ L4/L5 } & \multicolumn{2}{|c|}{ L5/S1 } \\
\hline & DTA & ESO & DTA & ESO & DTA & ESO \\
\hline FEM & 2,37 & 2,49 & 3,30 & 3,24 & 4,20 & 3,72 \\
\hline FEM & 2,37 & 2,58 & 2,97 & 3,05 & 3,93 & 3,57 \\
\hline FEM & 2,33 & 2,66 & 3,53 & 2,91 & 3,66 & 4,10 \\
\hline FEM & 2,08 & 2,47 & 2,74 & 3,00 & 4,97 & 4,20 \\
\hline FEM & 2,71 & 2,37 & 2,92 & 3,38 & 3,20 & 3,60 \\
\hline FEM & 2,62 & 2,28 & 3,64 & 3,43 & 4,43 & 4,12 \\
\hline FEM & 2,01 & 2,51 & 2,97 & 3,22 & 3,50 & 3,86 \\
\hline FEM & 2,47 & 1,79 & 2,67 & 2,49 & 2,81 & 2,62 \\
\hline FEM & 2,43 & 2,41 & 3,06 & 2,99 & 3,89 & 3,47 \\
\hline FEM & 2,21 & 2,70 & 3,49 & 3,85 & 3,64 & 4,66 \\
\hline FEM & 1,97 & 2,35 & 2,95 & 2,78 & 3,45 & 3,68 \\
\hline FEM & 1,68 & 2,28 & 2,92 & 2,88 & 3,74 & 3,99 \\
\hline FEM & 2,24 & 2,91 & 3,59 & 2,98 & 3,87 & 3,99 \\
\hline FEM & 2,77 & 2,67 & 3,14 & 3,59 & 4,17 & 4,22 \\
\hline FEM & 2,02 & 1,98 & 2,86 & 2,78 & 3,50 & 3,68 \\
\hline FEM & 2,20 & 2,32 & 2,80 & 2,84 & 3,75 & 3,86 \\
\hline FEM & 2,35 & 2,40 & 3,40 & 3,52 & 3,95 & 3,90 \\
\hline FEM & 2,30 & 2,42 & 2,98 & 2,90 & 3,80 & 3,83 \\
\hline FEM & 2,28 & 2,65 & 3,45 & 3,10 & 3,90 & 3,98 \\
\hline FEM & 2,25 & 2,35 & 3,10 & 3,00 & 3,80 & 3,90 \\
\hline FEM & 2,10 & 2,57 & 2,79 & 2,95 & 4,60 & 4,30 \\
\hline FEM & 2,10 & 2,60 & 2,90 & 3,10 & 3,60 & 3,79 \\
\hline FEM & 2,40 & 2,37 & 3,10 & 2,90 & 3,90 & 3,50 \\
\hline FEM & 2,25 & 2,38 & 2,85 & 2,95 & 3,85 & 3,95 \\
\hline FEM & 2,18 & 2,52 & 2,83 & 2,95 & 3,75 & 3,90 \\
\hline MAS & 2,79 & 2,89 & 3,47 & 3,42 & 3,99 & 3,59 \\
\hline MAS & 2,02 & 2,35 & 2,34 & 2,67 & 2,86 & 3,31 \\
\hline MAS & 2,63 & 2,32 & 3,47 & 3,12 & 3,77 & 3,66 \\
\hline MAS & 2,67 & 2,77 & 3,19 & 3,59 & 3,89 & 3,77 \\
\hline MAS & 2,30 & 1,93 & 3,14 & 2,20 & 3,49 & 2,93 \\
\hline MAS & 2,45 & 2,70 & 3,05 & 3,26 & 3,32 & 3,68 \\
\hline MAS & 1,89 & 2,03 & 2,70 & 2,47 & 2,68 & 3,24 \\
\hline MAS & 1,79 & 2,47 & 2,49 & 2,62 & 2,62 & 2,81 \\
\hline MAS & 2,08 & 2,12 & 3,36 & 3,89 & 4,08 & 3,49 \\
\hline MAS & 2,95 & 2,70 & 3,28 & 3,65 & 4,17 & 3,72 \\
\hline MAS & 1,78 & 2,14 & 2,58 & 2,59 & 3,18 & 3,07 \\
\hline MAS & 2,72 & 2,81 & 3,76 & 3,29 & 4,28 & 3,89 \\
\hline MAS & 2,71 & 2,52 & 3,29 & 3,39 & 4,07 & 3,91 \\
\hline MAS & 2,74 & 2,60 & 3,35 & 3,47 & 3,98 & 4,02 \\
\hline MAS & 2,50 & 2,57 & 3,52 & 3,12 & 3,97 & 3,81 \\
\hline MAS & 3,0 & 2,75 & 3,12 & 2,9 & 3,87 & 3,69 \\
\hline MAS & 2,78 & 2,84 & 3,45 & 3,78 & 4,1 & 3,99 \\
\hline MAS & 2,42 & 2,77 & 3,23 & 2,94 & 3,83 & 4,07 \\
\hline MAS & 2,62 & 2,65 & 3,08 & 3,75 & 3,6 & 3,91 \\
\hline MAS & 2,82 & 2,93 & 3,48 & 3,57 & 4,03 & 4,1 \\
\hline MAS & 2,13 & 2,05 & 2,96 & 2,88 & 3,75 & 3,85 \\
\hline MAS & 2,38 & 2,48 & 3,49 & 3,69 & 4,04 & 3,85 \\
\hline MAS & 2,34 & 3,0 & 3,69 & 3,32 & 3,97 & 4,05 \\
\hline MAS & 2,72 & 2,48 & 3,86 & 3,63 & 4,4 & 4,3 \\
\hline MAS & 2,67 & 1,99 & 2,75 & 2,69 & 3,1 & 3,35 \\
\hline
\end{tabular}


neurovasculares é manter a sua função a mais próxima do normal. A musculatura posterior serve como estabilizador da coluna lombar. O músculo multífidus é o primeiro grupo muscular responsável pela estabilização da junção lombosacra ${ }^{14,15}$. A abordagem convencional desinsere completamente este grupo muscular comprometendo sua inervação e suprimento sanguíneo, causando uma atrofia e retração cicatricial ${ }^{10,16}$. A perda dos estabilizadores e a alteração da mobilidade lombar podem contribuir para uma instabilidade e ser a causa de dores crônica ${ }^{10,12,16}$. Para o desenvolvimento e utilização das técnicas minimamente invasivas e necessário um conhecimento tridimensional da anatomia da coluna vertebral, ao contrário do que ocorre nos procedimentos convencionais onde há uma ampla exposição das estruturas, as cirurgias minimamente invasivas geralmente apresentam uma área cirúrgica limitada sendo fundamental o conhecimento anatômico. Procuramos utilizar a ressonância nuclear magnética um auxilio para um entendimento melhor da anatomia muscular posterior lombar. Dilatadores tubulares tem sido introduzidos recentemente para minimizar a agressão, porém a identificação das estruturas anatômica pode ser perdida e a necessidade de uma melhor exposição exige ressecção de elementos que deveriam ser preservados, em virtude disso preferimos o uso de lâminas como afastadores ${ }^{5}$. De fato, o entendimento anatômico e visualização é essencial para uma abordagem que visa minimizar os danos. Não encontramos na literatura descrição de um ponto de referência anatômico que indique a entrada entre o músculo multífidus e longuíssimo. A depressão que existe nos cortes axiais da ressonância nuclear magnética e que encontramos na abordagem cirúrgica indica o plano de clivagem entre o músculo longuíssimo e iliocostal. Para identificarmos o plano entre o músculo multífidus e longuíssimo é importante que a incisão da fáscia seja o mais próximo possível desse intervalo e esta medida pode ser conseguida pela identificação pré-operatório no exame de ressonância nuclear magnética, utilizando os cortes axiais, tanto em T1 como em T2, objeto deste trabalho. Verificamos que a distância mínima foi de $1,78 \mathrm{~cm}$ e a máxima de $5 \mathrm{~cm}$. No nível L3/L4 a média é de $2,42 \mathrm{~cm}$, no nível L4/L5 a media é de $3,13 \mathrm{~cm}$ e no nível L5/S1 é de $3,77 \mathrm{~cm}$, aumentando a distância média no sentido craniocaudal nos níveis lombares inferior. Nossos resultados foram semelhantes ao estudo de Vialle et al. ${ }^{17}$ que aferiram a distância da linha media ao plano intermuscular entre os músculos multífidus e longuíssimo através da dissecção em 30 cadáveres e encontraram uma distância média de $4 \mathrm{~cm}$ na altura do processo espinhoso de $L 4$, variando de $2,4 \mathrm{~cm}$ a $5,5 \mathrm{~cm}$. Os resultados cirúrgicos dependem de uma série de fatores. O conhecimento anatômico por parte dos cirurgiões é fundamental, principalmente se procurarmos diminuir a lesão aos tecidos, então entendemos que o exame de ressonância nuclear magnética acrescenta informações importantes para o planejamento préoperatório dessa via de abordagem.

\section{CONCLUSÃO}

Entendemos que para execução da cirurgia minimamente invasiva na coluna lombar é fundamental a identificação do plano intermuscular entre os músculos multífidus e longuíssimo. O uso da ressonância nuclear magnética no planejamento pré-operatório foi essencial para localização desse plano. Concluímos neste estudo que a distância da linha média até o plano intermuscular foi de 2,42 $\mathrm{cm}$ a 3,77 cm em média e que ela aumenta no sentido craniocaudal nos níveis lombares inferiores (L3/L4,L4/L5 e L5/S1). Não encontramos diferença estatisticamente significativa entre as medidas quando comparamos os sexos nos níveis L4/L5 e L5/S1, portanto, o planejamento pré operatório não sofre influencia dos sexos.

\section{REFERÊNCIAS}

1. Mathieu P, Demirleau J. Surgical therapy in painful spondylolisthesis. Rev Chir Orthop. 1936;23:352

2. Watkins MB. Posterolateral bonegrafting for fusion of the lumbar and lumbosacral spine. J Bone Joint Surg Am. 1959:41(3):388-96.

3. Wiltse LL, Hutchinson RH. Surgical treatment of spondylolisthesis. Clin Orthop Relat Res. 1964;35:116-35.

4. Wiltse LL, Spencer CW. New uses and refinements of the paraspinal approach to the lumbar spine. Spine (Phila Pa 1976). 1988;13(6):696-706

5. Hoh DJ, Wang MY, Ritland SL. Anatomic features of the paramedian musclesplitting approaches to the lumbar spine. Neurosurgery. 2010;66(3Suppl Operative):13-24

6. Macintosh EJ, Valencia F, Bodguk N, Munro RR. The morphology of the human lumbar multifidus. Clin Biomech. 1986:1:196-204.

7. Foley KT, Holly LT, Schwender JD. Minimally invasive lumbar fusion. Spine (Phila Pa 1976). 2003:28(15 Suppl):S26-35

8. Kawaguchi $Y$, Matsui $H$, Tsuji $H$. Back muscle injury after posterior lumbar spine surgery. A histologic and enzymatic analysis. Spine (Phila Pa 1976). 1996;21(8):941-4

9. Kawaguchi $Y$, Matsui $H$, Tsuji H. Back muscle injury after posterior lumbar spine surgery. Part 2: Histologic and histochemical analyses in humans. Spine (Phila Pa 1976). 1994;19(22):2598-602.
10. Gejo R, Matsui $H$, Kawaguchi $Y$, Ishihara $H$, Tsuji $H$. Serial changes in trunk muscle performance after posterior lumbar surgery. Spine (Phila Pa 1976). 1999;24(10):1023-8.

11. Styf JR, Willén J. The effects of external compression by three different retractors on pressure in the erector spine muscles during and after posterior lumbar spine surgery in humans. Spine (Phila Pa 1976). 1998:23(3):354-8.

12. Mayer TG, Vanharanta H, Gatchel RJ, Mooney V, Barnes D, Judge L, et al. Comparison of CT scan muscle measurements and isokinetic trunk strength in postoperative patients. Spine (Phila Pa 1976). 1989:14(1):33-6.

13. Rantanen J, Hurme M, Falck B, Alaranta H, Nykvist F, Lehto $M$, et al. The lumbar multifidus muscle five years after surgery for a lumbar intervertebral disc herniation. Spine (Phila Pa 1976). 1993:18(5):568-74.

14. Donisch EW, Basmajian JV. Electromyography of deep back muscles in man. Am J Anat. 1972;133(1):25-36.

15. Hansen L, de Zee M, Rasmussen J, Andersen TB, Wong C, Simonsen EB. Anatomyand biomechanics of the back muscles in the lumbar spine with reference to biomechanical modeling. Spine (Phila Pa 1976). 2006;31(17):1888-99.

16. Sihvonen T, Herno A, Paljärvi L, Airaksinen O, Partanen J, Tapaninaho A. Local denervation atrophy of paraspinal muscles in postoperative failed back syndrome. Spine (Phila Pa 1976). 1993:18(5):575-81.

17. Vialle R, Court C, Khouri N, Olivier E, Miladi L, Tassin JL, et al. Anatomical study of the paraspinal approach to the lumbar spine. Eur Spine J. 2005;14(4):366-71. 